\title{
The Power of Emotional Intelligence
}

\author{
Swapnil Ahuja \\ Information Technology \\ Guru Gobind Singh Indraprastha University, \\ Sector 16 C, Dwarka, New Delhi, DL 110078
}

\begin{abstract}
In this paper various aspects of emotional intelligence would be reviewed. Present systems would be discussed along with their better implementation using Brain Emotional Learning. We would also propose the unconventional applications of emotional intelligence that would enable us to integrate these in present for more powerful application than present now. This paper also predicts the change that will happen once we start using these systems having emotional intelligence.
\end{abstract}

\section{Keywords}

Emotional Learning, Emotional Intelligence, BELBIC, Human Interaction, Emotional Attachment, Emotional Intelligence Applications

\section{INTRODUCTION}

Artificial Intelligence has been one of the most widely discussed topic in computer science or electronics since its introduction back in 1956.Emotional intelligence is a very important part of Artificial Intelligence as need of emotionally intelligent machines is required to take the level of computing to the next level or to make it replicate the thinking process of human brain rather than using primitive methods of spoon feeding the machine. Emotions are what that separate humans from others and due to this reason our decisions may sometimes sound logically incorrect which leads to unpredictable nature of humans which is not possible in computers ,even random generated numbers are not truly random as they are also following some algorithm. This would introduce unpredictable nature which can be of great importance in some cases.

We first start by analyzing the present systems and their weaknesses and then how can better implementation could take place using BELBIC (Brain Emotional Learning Based Intelligent Controller) suggested by Caro Lucas.

\section{PRESENT SYSTEMS}

There are a lot of systems using emotional intelligence few of them are given below

\subsection{Automatically Recognizing Facial Expression}

Its system uses CERT (Computer Expression Recognition Toolbox) Technology [1] which can track frame by frame various facial expressions. It is used during tutoring students studying their engagement or when they are getting bored during their learning process. It is aimed to be used widely for a variety students using MOOC (Massive Open Online Course) so that it could provide us meaningful data.

\subsection{Beyond Verbal}

An Israeli Startup [2] that analysis human emotion just based on their raw voice as they speak in real time. It's far effective of emotion analytics than all traditional methods which tried to achieve the same thing. It also provides a cloud based system through which anybody can use this service according to their need .It is the first step into the future but not even near to the capability of emotional intelligence.

\subsection{Apple Siri [3] /Google Now[4]}

Both are not exactly examples of using emotional intelligence but they are mentioned here because there is a lot of scope of using it in these technologies and these are usually reach more customers than any of the above mentioned. Still we see they are lot of problems in understanding a particular user's needs because it uses simple speech to text conversion and only understands literal meanings which we can overcome easily by emotional intelligence.

\subsection{Nao Robot[5]}

It was the first robot to have emotional intelligence not fully but to a far greater extent than the world had ever seen. It could interact with humans, feel sad, scared and could even understand people's emotions that it met regularly.

Out of all the above the Nao Robot is closest to how emotional intelligence could actually be used whereas all the other three are basically devices or software that are programmed to perform a particular task. Let us take the first case where it only detects human emotions but not actually understands emotions itself and then react according to it in particular way. It's just a system that if the face is like this then it is a particular emotion, it cannot self interpret the various emotions. In the second case it predicts emotions based on our voice but does not learn anything from it and react differently or improve over time like humans.

\section{IMPLEMENTATION}

We can implement emotional intelligence by the method of machine learning. Machine Learning is the process of letting the machine learn by itself rather than guiding or programming the machine for everything or every task it has to perform. It's a very powerful concept of artificial intelligence which is being used for over the last few decades but its use is not popular in the field of emotional intelligence.

\subsection{Brain Emotional Learning}

This model was developed by Moren and Balkenius [7] that depicts the learning process of brain by typically describing those parts that deal with emotions in the human brain such as Amygdala, Orbitofrontal Cortex, Thalamus, Sensory Input Cortex etc. The model is depicted in the figure 1 below 


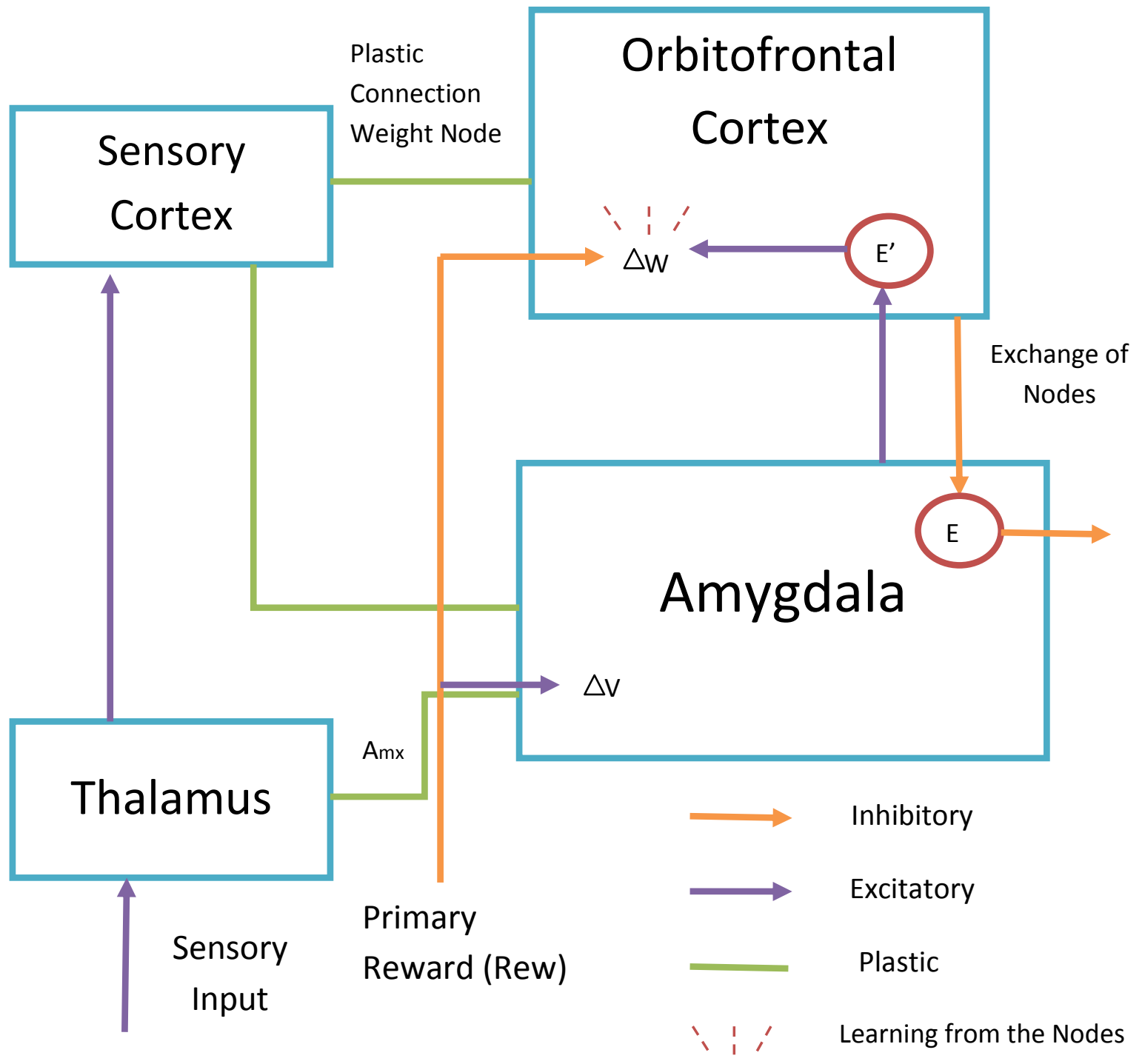

Fig.1 Graphical depiction of the Brain Emotional Learning (BEL) process [9]

The Model is divided into mainly two parts: Amygdala and Orbitofrontal Cortex[7]. The purpose of Primary Reward is still not very clear[7].

\subsection{Brain Emotional Learning Based Intelligent Controller(BELBIC)}

It's a controller proposed by Caro Lucas [10] and based on the model mentioned above developed by Moren And Balkenius which is used to mimic those parts of the brain that are used to produce emotions. In our body Limbic system is responsible for emotional learning and the power of emotional learning is far greater than the gradient or traditional learning algorithms due to their high level of complexities, therefore BELBIC is the effective model to adopt for creating emotionally intelligent machines. Emotional Intelligence can build upon the concepts of machine learning. Let us take the case of the Face Recognition Application developed by the North Carolina State University. Its main purpose was to track students studying and detect or analyze whether they are bored or frustrated or engaged in active learning and it detects by scanning our facial expressions using a camera. Now the machine knows that how humans react if they are feeling a particular emotion and also what their facial structure is, either the eyebrow is raised or not. Therefore this all can account for a dataset for a machine and we can let the machine learn with help of machine learning algorithm .This leads us taking the step forward from just by using data that we have collected can be efficiently used for the machine to gain knowledge and finally developing some type of emotional intelligence.

Even Apple's Siri or Google Now can be developed on which they can learn with period of time about their user and slowly form a bond with user according to their needs. In further sections we would throw light on how all this can be beneficial in practical applications.

\section{APPLICATIONS}

Emotion is an important aspect of human behavior and this reflects in our decision making as we cannot define everything in yes/no or true/false as a machine or computer does. Usually people associate emotional intelligence with robots but it can open many other windows for us in various fields as suggested below:

\subsection{Treatment of Psychotic Patients and Prevent Suicides}

Large number of applications for smart phones can be developed that track the activity of user then we can study about the behavior or usage pattern to understand how a 
person reacts just before a suicide and ultimately develop an application that can prevent the person from giving away his life and a machine can only achieve this if it has some kind of emotional connect with the person because one of the major factors leading to suicide are emotional tensions or person doesn't have anybody to whom he can talk and this also can be used in treatment of many patients suffering from psychotic depressions.

\subsection{Better Process Scheduling}

There are large number of process scheduling algorithms [11] developed for assigning priority to various processes but these algorithms does not involve the user in anyway but after introducing emotional intelligence in process scheduling we could prefer or prioritize process based on user's present condition .Let us take an example if a person is in a tensed or atrocious situation and his primary task to make an emergency call but processor in the phone decide to complete some other task which is provided the time slice at that moment and puts the calling task in the waiting queue and if the user forces it to switch the call cannot be made as now it gets hanged but if the phone could understand the user's situation it could preempt all other running processes and then could easily continue with dialing the call. It maybe life saving in some situations.

\subsection{Better Search Results/Recommender System}

Recommender systems of products on online stores are very rigid or even static in comparison to human brain, the way it thinks. Most people take decisions during online buying which maybe logically not so right but emotionally feels correct such as gifting someone something expensive. Now if machines start understanding human emotions they would recommend far better products than now which are just static suggestions based on a few parameters such as price or color. Search Results would be far more accurate after they are emotionally aware of the user whose is performing the search.

\subsection{More Secure Systems}

Systems could easily differentiate between malicious and genuine users far more effectively as humans could easily identify people and it is difficult to con a person in comparison to computer machine but if programmed incorrectly it could also lead to situations in which emotions could pose as a disadvantage.

\section{HUMAN INTERACTION}

Human interact with other human in a different in comparison to their gadgets or computer because the presence of emotions. However humans still express emotions about certain things such either they like a mobile phone or not, but this not the other way around. This would change as we infuse emotional intelligence in these products, their interaction with humans will change significantly and also humans would now look at them differently.

The Leap (Leveraging Emotional Attachment for Profit) Index [12] by New Media Matrix gives us insight into the way consumers feel about products even when they are not much interactive than they will post the introduction of emotions in them.(Every year Leap Index is given by New Media Matrix and Leap Index is their reserved trademark).

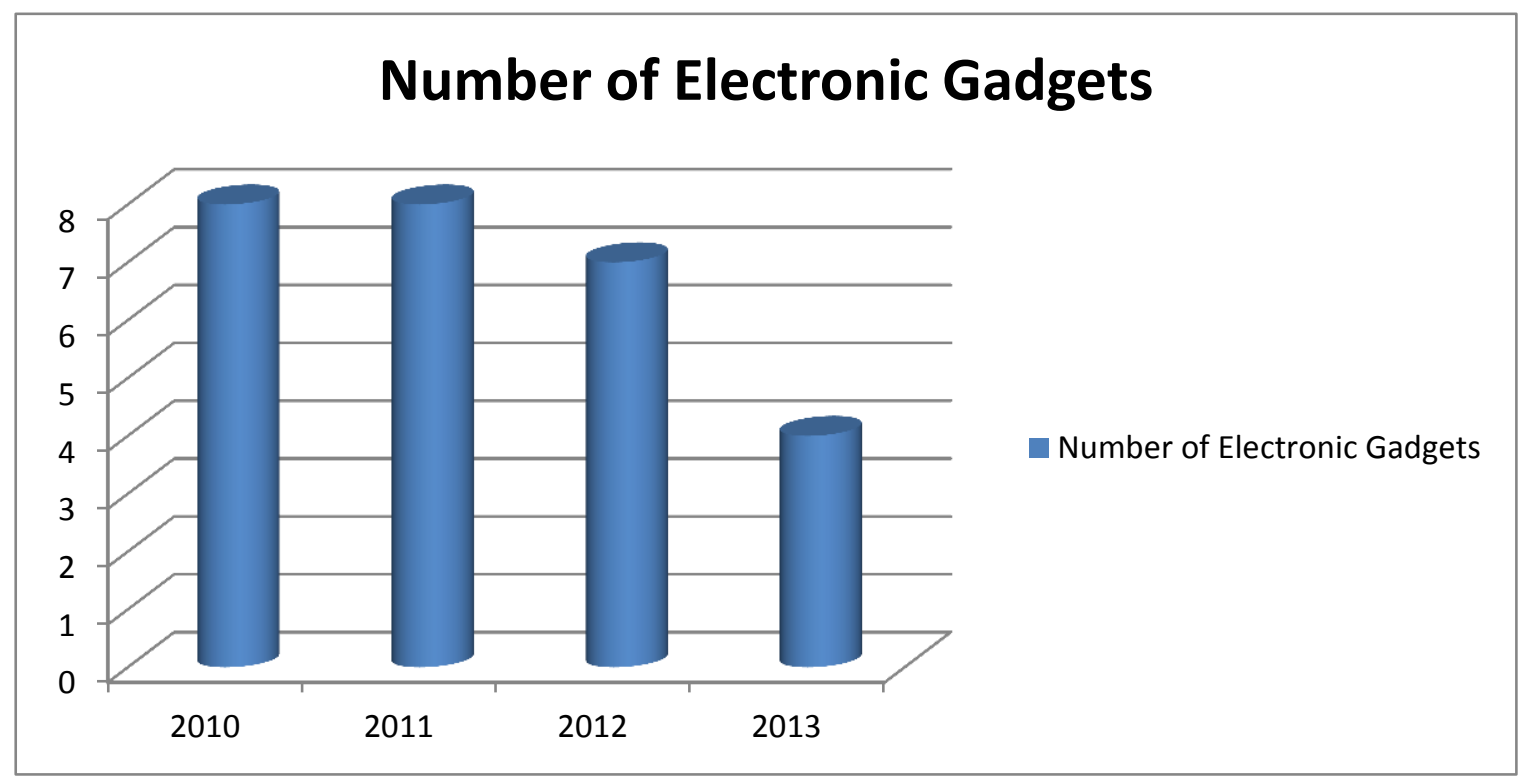

Fig 2 Number of Electronic Gadgets Vs Year (Based on Leap Index) [12]

Looking the statistics from $2010-2013$ as given by the leap index [12], majority brands that deals with personal gadgets always in the top 10 of the leap index leaving away 2013 in which it fell drastically low to only 4 .This also indicates that gradual fall in the leap index for the personal gadgets is the indication that now consumers have become habitual to these things and do not care about them emotionally.Still a consistent place in the top 10 is reserved by the apple products and the company itself which only featured at 13 in the year 2011 only. Majority of products in the top 10 are iPod, iPhone, iPad which indicates the high emotional attachment of consumers towards apple and the decision to buy these is to far greater extent emotional.

This information is useful for the big tech giants that after the introduction of emotions in their products, consumers could not be solely attracted on specifications of product but also the past experience it had with product both emotionally and physically while using it. Consumers may also become reluctant to change their products for a newer one as it is always to difficult make a new friend. 


\section{CONCLUSION}

In this paper we have shown that the present systems that said to include emotional intelligence are still at infancy stage but everything starts at by taking small baby steps. The BELBIC (Brain Emotional Learning Based Intelligent Controller) suggested by Caro Lucas is effective in implementing emotional learning. The human N\nature would also change along with change in systems as they may become physically, mentally and emotionally dependent on these systems. But still the BELBIC has its share of weaknesses that could overcome in the future.

\section{REFERENCES}

[1] Joseph F. Grafsgaard ${ }^{1}$, Joseph B. Wiggins ${ }^{1}$, Kristy Elizabeth Boyer ${ }^{1}$, Eric N. Wiebe ${ }^{2}$,James C. Lester ${ }^{1}$ ,Automatically Recognizing Facial Expression: Predicting Engagement and Frustration, Department of Computer Science ${ }^{1}$,Department of STEM Education ${ }^{2}$ ,North Carolina State University, Raleigh, NC, USA

[2] Beyond Verbal Website [Online]. Available : http://www.beyondverbal.com/

[3] Apple Siri Website[Online].Available: https://www.apple.com/ios/siri/

[4] Google Now Website[Online].Available: http://www.google.com/landing/now/

[5] Article on Nao Robot from The Guardian Website [Online]. Available: http://www.theguardian.com /technology/2010/aug/09/nao-robot-develop-displayemotions/
[6] Nicholas L. Cassimatis, Human-Level Artificial Intelligence Must Be an Extraordinary Science, Advances in Cognitive Systems 137 -45,(July 2012)

[7] Zahra Beheshti, Siti Zaiton Mohd Hashim, A Review of Emotional Learning And It's Utilization in Control Engineering, Int. J. Advance. Soft Comput. Appl., Vol. 2, No. 2, (July 2010)

[8] H. Zhang, S. Liu and S. Y. Yang, An emotion-based behavior coordination approach to autonomous mobile robots, Proceedings of the International Conference on Sensing, Computing and Automation, Vol. 1, (2006)

[9] Ehsan Lotfi, A simple Mathematical Fuzzy Model of Brain Emotional Learning to Predict Kp Geomagnetic Index,IJISAE(2014)

[10] C. Lucas, D. Shahmirzadi, N. Sheikholeslami, Introducing BELBIC: Brain Emotional Learning Based Intelligent Controller, International Journal of Intelligent Automation and Soft Computing, pp. 11-22, Vol. 10, NO.1, (2004)

[11] Prerna Ajmani , Manoj Sethi, Proposed Fuzzy CPU Scheduling Algorithm (PFCS) for Real Time Operating Systems, BIJIT - 2013; ; Vol. 5 No. 2, (July-December 2013)

[12] Leap Index by New Media Metrics [Online].Available: http://www.newmediametrics.net/splash/services-studiesleap.aspx 